\title{
ANALYSIS OF EFFECTIVENESS OF PHOENIX ENTRY REACTION CONTROL SYSTEM
}

\author{
Artem A. Dyakonov, ${ }^{*}$ Christopher E. Glass ${ }^{\dagger}$ and Prasun N. Desai ${ }^{\ddagger}$ \\ NASA LaRC, Hampton, VA, 23681, USA \\ John W. Van Norman ${ }^{\S}$ \\ Analytical Mechanics Associates, Hampton, VA, 23666, USA
}

\begin{abstract}
Interaction between the external flowfield and the reaction control system (RCS) thruster plumes of the Phoenix capsule during entry has been investigated. The analysis covered rarefied, transitional, hypersonic and supersonic flight regimes. Performance of pitch, yaw and roll control authority channels was evaluated, with specific emphasis on the yaw channel due to its low nominal yaw control authority. Because Phoenix had already been constructed and its RCS could not be modified before flight, an assessment of RCS efficacy along the trajectory was needed to determine possible issues and to make necessary software changes. Effectiveness of the system at various regimes was evaluated using a hybrid DSMC-CFD technique, based on DSMC Analysis Code (DAC) code and General Aerodynamic Simulation Program (GASP), the LAURA (Langley Aerothermal Upwind Relaxation Algorithm) code, and the FUN3D (Fully Unstructured 3D) code. Results of the analysis at hypersonic and supersonic conditions suggest a significant aero-RCS interference which reduced the efficacy of the thrusters and could likely produce control reversal. Very little aero-RCS interference was predicted in rarefied and transitional regimes. A recommendation was made to the project to widen controller system deadbands to minimize (if not eliminate) the use of RCS thrusters through hypersonic and supersonic flight regimes, where their performance would be uncertain.
\end{abstract}

\section{Nomenclature}

$C_{p} \quad$ Pressure coefficient

$C_{X} \quad$ Moment coefficient

$K n D \quad$ Knudsen number based on diameter

$L \quad$ Length, also moment arm, m

$M \quad$ Mach number, also moment, $\mathrm{N}-\mathrm{m}$

$R C S$ Reaction Control System, also used for roll thrusters

$S \quad$ Area, $\mathrm{m}^{2}$

TCM Trajectory Correction Maneuver, also used for pitch-yaw thrusters

$T$ Torque, $\mathrm{N}-\mathrm{m}$

$v \quad$ velocity, $\mathrm{m} / \mathrm{sec}$

$\alpha \quad$ Angle of attack, degrees

$\beta \quad$ Angle of sideslip, degrees

$\chi \quad$ Mole fraction

$\rho \quad$ Density, $\mathrm{kg} / \mathrm{m}^{3}$

Subscript

$\infty \quad$ Free-stream

*Aerospace Engineer, Atmospheric Flight Entry Systems Brach, AIAA Member

$\dagger$ Aerospace Engineer, Aerothermodynamics Branch, AIAA Senior Member

${ }^{\ddagger}$ Senior Aerospace Engineer, Atmospheric Flight Entry Systems Branch, AIAA Associate Fellow

$\S$ Senior Project Engineer, Atmospheric Flight Entry Systems Branch, AIAA Member 


\section{Introduction}

On May 25, 2008 Phoenix successfully landed on Mars. Phoenix entered the Martian atmosphere directly from its interplanetary trajectory and executed a ballistic three-axis stabilized non-spinning entry into the atmosphere shedding its initial energy to levels appropriate for a safe landing. Phoenix is the first Mars mission to execute a non-spin-stabilized entry from such high velocity; all successful missions before Phoenix employed different strategies. In 1976, Vikings 1 and 2 performed controlled unguided entries from circular orbit, whereas more recent missions, namely Pathfinder (1997), and Mars Exploration Rovers (2004) entered directly on interplanetary trajectories but utilized spin-stabilization. Because the next Mars mission, Mars Science Laboratory (MSL), is designed to fly a guided lifting entry from an interplanetary approach, the flight experience of Phoenix is very valuable.

As the Phoenix capsule (see Fig. 1) entered the atmosphere, it interacted with the surrounding atmospheric gas. This interaction produced aerodynamic forces and moments that acted on the vehicle during entry, and in the process reduced capsule's energy to an acceptable value for the deployment of the parachute. Variations in atmospheric properties and aerodynamics of the capsule during entry can produce pitch and yaw oscillations. To control these oscillations and to perform a final azimuthal alignment, the Phoenix entry capsule was equipped with a Reaction Control System (RCS) designed to enable rate damping and roll control during entry. The RCS is composed of hydrazine thrusters capable of generating control torques, fuel tankage, and control valves operated by the flight control program.

During the course of the atmospheric entry the RCS thrusters would be fired to produce torques about the pitch, yaw and roll axes, commanded by the control program. The control program may issue commands at any time during entry. Therefore, the control system must be effective in all regimes from rarefied to supersonic, where parachute deployment occurs. The RCS thrusters fire into the capsule's wake. During operation, the thruster effluent interacts with the wake and alters it. Because pressure on the backshell of the capsule is not zero, interactions between thruster plumes and the capsule wake can cause a change in the aftbody pressure distribution. One possible result is the emergence of capsule moments that may compete with the native authority of the control system. It is possible to generate aftbody moments that create gain in a given channel or cross-coupling into other channels. An example of gain would be if in response to activation of pitch thrusters the capsule would develop an aerodynamic pitching moment, that would add or subtract from the native RCS moment. An example of cross-coupling would be if in response to activation of roll thrusters, the capsule would develop some pitch moment, such that pitch thrusters would have to be used to counter it.

\section{Background}

Effects of RCS-wake interaction depend greatly on the details of the local flow in the vicinity of the thruster exit. Properties of the local flow depend on the free-stream parameters, atmospheric composition, capsule size, shape and attitude and the location of the thruster. The local flow can be part of the attached wake, in which case its typically supersonic, or it can be a part of separated wake, in which case it is typically subsonic. Under the influence of some RCS thrusters, a separated wake may be forced to reattach, whereas an attached wake may be forced to separate. Generally, attached flow is more energetic, and interactions between it and the thruster effluent can produce shock structures referred to as horse-shoe shocks. Such structures develop a quazi-nozzle-like flow directed toward the surface, essentially creating a high energy stagnation flow at the surface of the capsule upstream of the thruster exit. This type of an interaction can result in a significant increase over the baseline in heating, pressure, and shear at the surface. Irrespective of the character of the local flow, any interaction between thruster effluent and local flow will result in changes to the wake. This is due to the fact that much of wake is subsonic, and changes in any given location affect any other location that is within the elliptical boundary. The result of this kind of dependence is that changes in surface environments can occur over most of the rear wall of the capsule when an RCS thruster is fired. Most of the environmental changes that occur within the separated part of the wake are small, however, any interaction with energetic flow outside of the wake shear layer, like the kind that will happen if the plume from the thruster nozzle punches through the separated zone and into more energetic flow, can result in significant changes in surface environments. Specifically, a change in surface pressure distribution will produce moments on the capsule, which can interfere with the native authority of the RCS.

The current approach to analysis of RCS-induced control interference is to use state of the art numerical 
techniques for flight predictions at flight conditions, and to use ground test facilities for validation. Because of time constraints, it was not possible to develop an experimental program to support the analysis of the efficacy of the Phoenix RCS. As such, the present analysis methodology relied entirely on computational techniques, while some validation was provided through ground testing of RCS effects by the Mars Science Laboratory (MSL) Project.

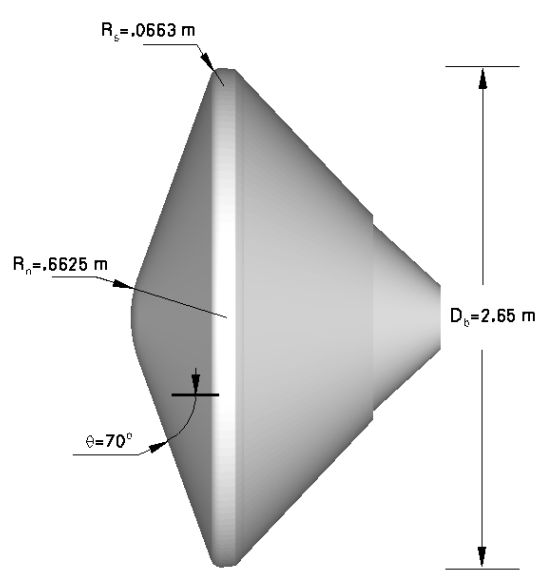

Figure 1. Phoenix capsule geometry

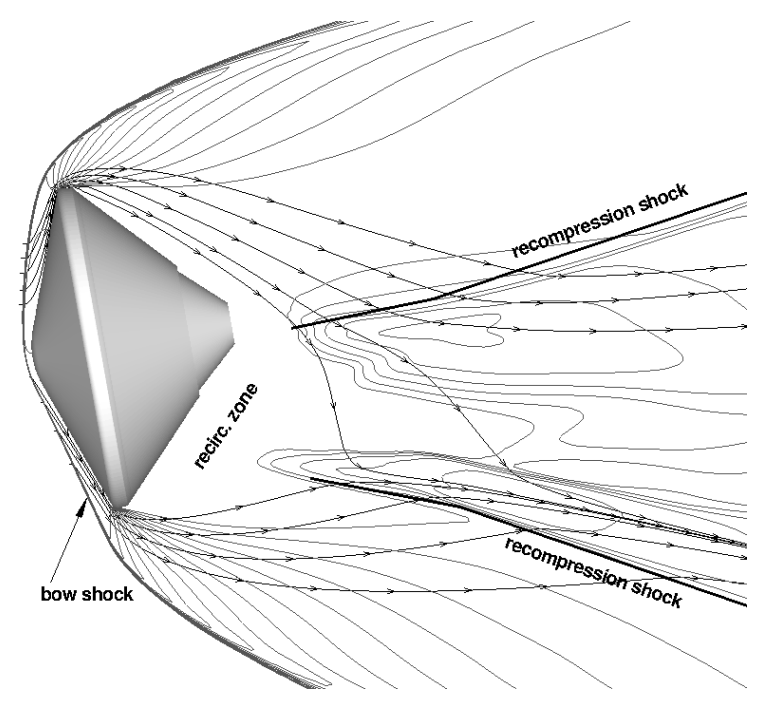

Figure 2. Illustration of the flowfield around Phoenix capsule

Because RCS thrusters exit into complex wake flow (Fig. 2), they induce diverse flow interaction phenomena. While these interactions may alter both the aerodynamic characteristics and aerothermodynamic environment of the capsule, this paper will specifically focus on analysis of the former (i.e. the induced aerodynamic moments). The objective of the present analysis is to determine the cumulative effect of changes in the basecover pressure distribution on the RCS control authority.

\section{Phoenix RCS}

Phoenix was designed to fly a ballistic three-axis stabilized trajectory. Trim angle of attack is near zero for most conditions, except when bounded instability occurs (see, for example, Gnoffo, ${ }^{1}$ Edquist $^{2}$ ). The RCS in this scenario is used mainly as a rate damper. The Phoenix RCS consists of four $26.3 \mathrm{~N}$ thrusters (TCM 1, 2, 3 and 4), used for pitch and yaw corrections and four $5.7 \mathrm{~N}$ thrusters (RCS 1,2, 3 and 4), providing roll authority. Layout is shown in Figs. 3 and 4. Figures 5 and 6 identify thruster firings to obtain positive pitching and yawing moment. Because of a small moment arm, the yaw thrusters (Figure 6) provide control authority of only $10.5 \mathrm{Nm}$ as opposed to $57 \mathrm{Nm}$ for the pitch thrusters (Fig. 5).

\section{Method}

\section{A. Overview}

The objective of the present analysis is to determine the magnitude of aerodynamic moments, developed on the aftbody of Phoenix entry capsule because of the interaction of RCS thruster plumes with the wake. The interference torque is defined as:

$$
M_{\text {interference }}=C m_{\text {interference }} \cdot S_{\text {ref }} \cdot L_{r e f} \cdot \frac{1}{2} \rho v^{2}
$$

where

$$
C m_{\text {interference }}=C m_{T C M}-C m_{\text {Baseline }}
$$

$C m_{T C M}$ is the aerodynamic moment on the capsule, whose surface pressure distribution is perturbed by presence of the thruster plumes. $\mathrm{Cm}_{\text {Baseline }}$ is the aerodynamic moment on the capsule in the baseline flow 


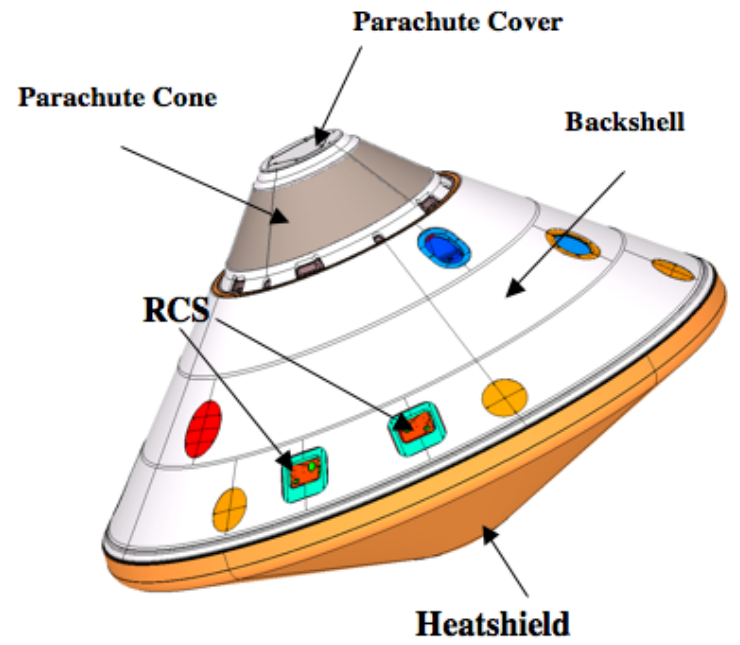

Figure 3. Phoenix capsule features

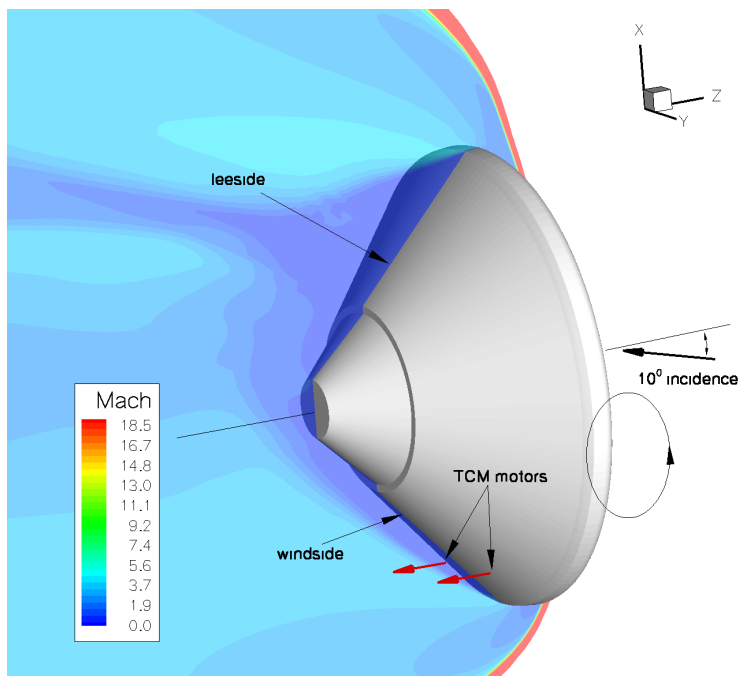

Figure 5. Pitch firing configuration

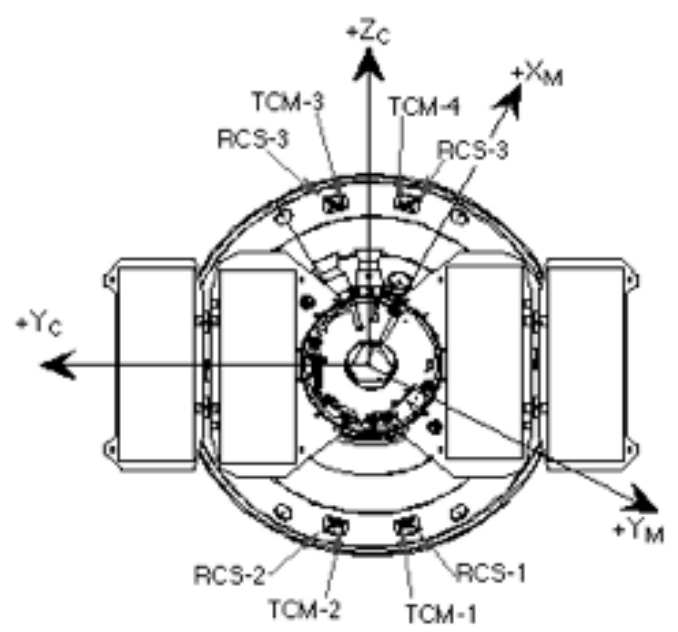

Figure 4. Phoenix RCS layout

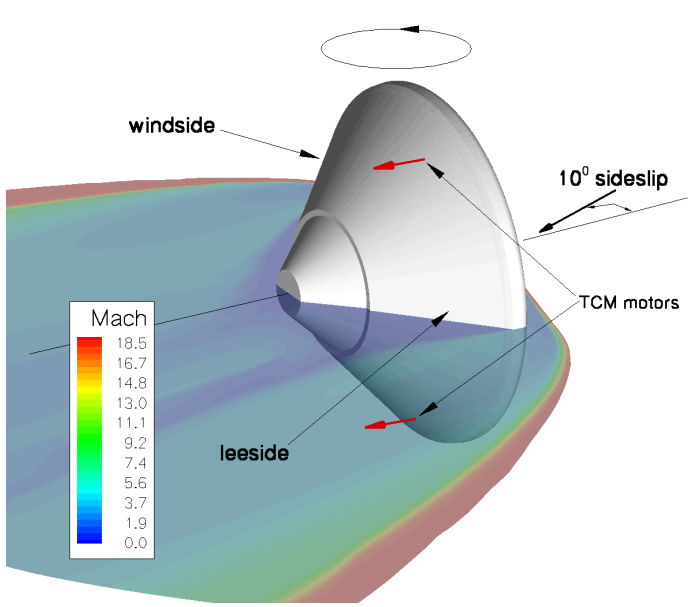

Figure 6. Yaw firing configuration

unperturbed by the thruster. Note, that torque due to thrust of the nozzles does not enter the definition of the interference moment. However, we can use it to define control gain:

$$
\text { Gain }=\frac{T_{T C M}+M_{\text {interference }}}{T_{T C M}}
$$

When the gain is less then unity, interference torque is creating a deficit of authority. When the gain is greater then unity, a surplus of authority is caused by the interference torque.

Figures 7 and 8 show acreage distribution of the available moment arm about yaw and pitch axes for the Phoenix aftshell. Moment arms are computed about the center of mass of the capsule. Plots indicate that there are areas, typically near capsule maximum diameter and near parachute cone, where the moment arm takes on a large positive or negative value. If surface pressure in one of those areas was altered due to the interaction of thruster and wake, an appreciable moment would be developed.

Present analysis of the RCS-induced interference effects covered the entire entry from rarefied to supersonic regime. Calculations were performed at a rarefied flow condition, corresponding to $\mathrm{KnD}=0.1$, at high hypersonic condition of Mach 27.2 (which corresponds to a $\mathrm{KnD}=0.0005$ ), at hypersonic Mach 18.8 (which 


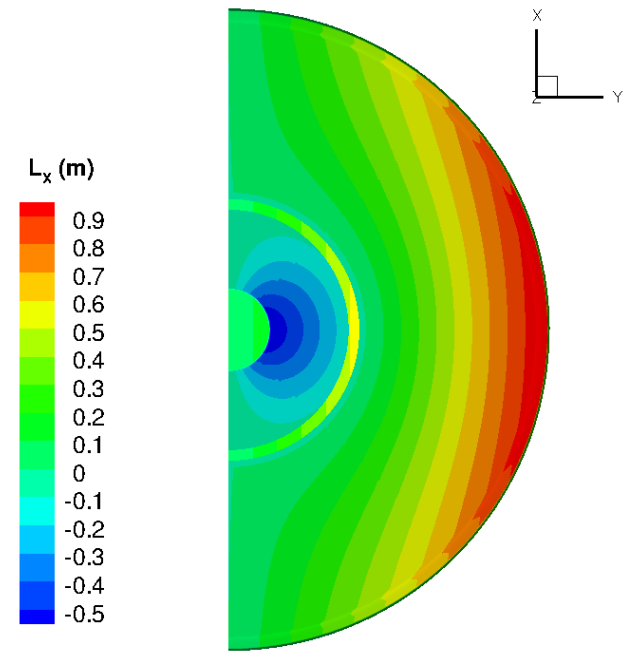

Figure 7. Moments about $\mathrm{X}$-axis

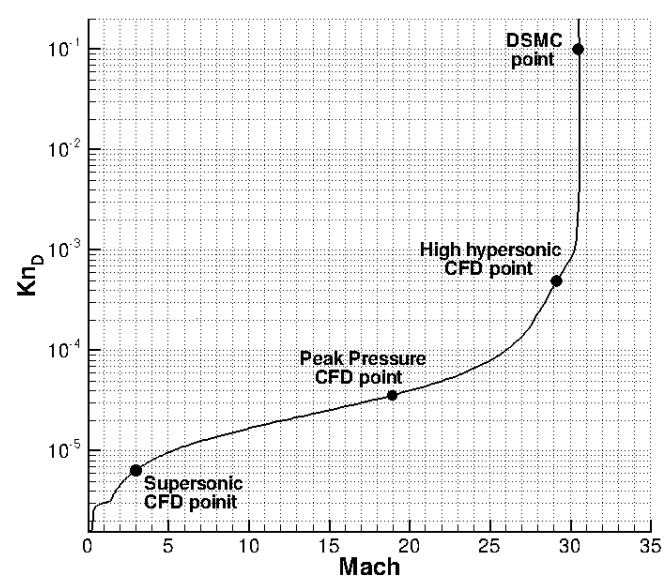

Figure 9. Investigated conditions
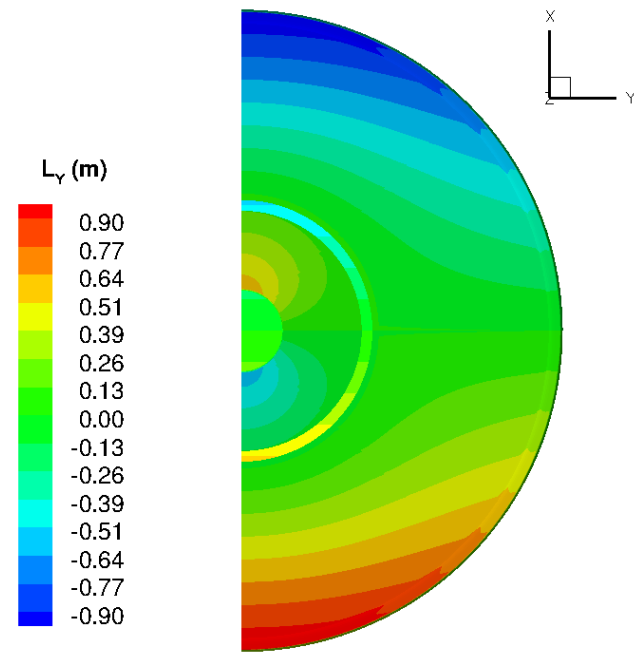

Figure 8. Moments about $\mathrm{Y}$-axis

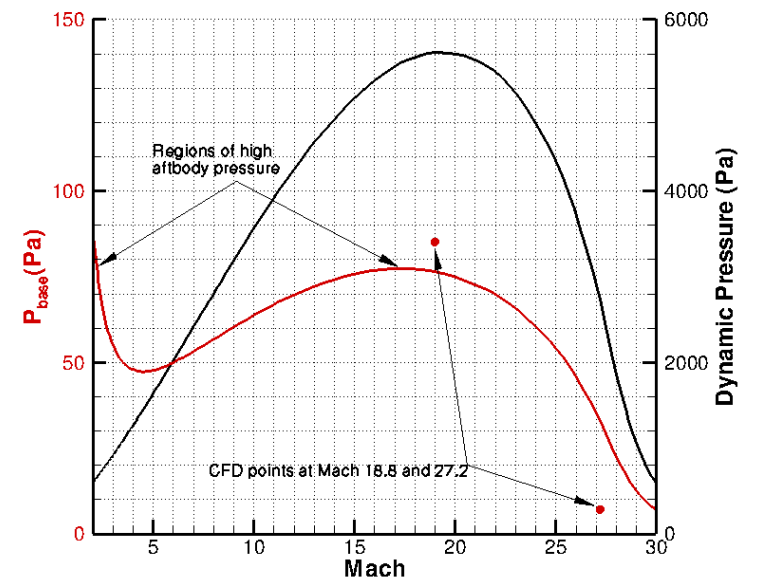

Figure 10. Variation of dynamic pressure and basecover pressure

is roughly peak dynamic pressure on Phoenix entry trajectory), and at supersonic Mach 3. These points are shown in the Fig. 9. Mach 18.8 was selected because the aftbody pressure goes through a maximum near peak free-stream dynamic pressure. Mach 3 condition was selected because of relatively high expected RCS activity during supersonic flight (due to a dynamic instability ${ }^{2}$ ) and because of a large contribution to the overall capsule moments expected from the aftbody. Aftbody pressure can be estimated from a base correction curve developed for Pathfinder by fitting Viking flight data: ${ }^{10}$

$$
C_{p, b}=a_{0}+\frac{a_{1}}{M_{\infty}}+\frac{a_{2}}{M_{\infty}^{2}}+\frac{a_{3}}{M_{\infty}^{3}}
$$

where $a_{0}=8.325 E-3, a_{1}=1.1293 E-1, a_{2}=-1.801$ and $a_{3}=1.2885$. Equation (4) can be solved for pressure on the basecover as a function of Mach number on a representative Phoenix design entry trajectory. Figure 10 shows the variation of dynamic pressure with Mach number and shows variation of basecover pressure, as computed from Eq. (4). The two points on the plot are CFD-predicted pressures. Generally, CFD follows the trend of the curve, but magnitudes don't always agree. Notably, the plot of base pressure indicates two regions where aftbody pressures peak. One of these regions is near peak dynamic pressure on 
the trajectory and another occurs during supersonic flight. These are the regions of most interest from the point of view of aero-RCS interactions.

A rarefied regime condition was selected to confirm that RCS interference at this early stage in entry should not be significant. The Mach 27.2 condition was selected to verify qualitative agreement with results of analysis in the rarefied regime.

Neither analysis attempted to simulate pulsed operation of thrusters. Because of the limited time accuracy of codes and high computer cost this was thought impractical. It is believed that the current set of simulations with a continuous thruster firing should be bounding of the expected phenomena.

\section{B. Rarefied Regime}

An analysis at a rarefied flow condition $\left(\mathrm{KnD}=0.1, \beta=-6^{\circ}\right)$ was performed to determine if there is an interaction between the thruster plumes and the aerodynamic flowfield. Because the flow field contains both a high pressure thruster, which expands into a rarefied external flow, the flow is split into two regions: the continuum region from the thruster stagnation to a continuum breakdown and the rarefied region outside the continuum flow. Computation Fluid Dynamics (CFD) is applied to the continuum region using the GASP code. ${ }^{3}$ GASP provides a stable CFD solution from the chamber to the expanding flow outside the reaction control system (RCS) thruster. The three-dimensional flow solution was performed on a 19-block, 3.14 million cell gridded from the thruster nozzle stagnation chamber through a converging-diverging throat. Inflow gas to the thruster chamber was modeled as partially dissociated, catalyzed hydrazine with mole fractions of $\chi(\mathrm{NH} 3)=0.37, \chi(\mathrm{N} 2)=0.27$, and $\chi(\mathrm{H} 2)=0.36$. For the CFD, the back shell wall was treated as a slip boundary, and the outflow boundary as a vacuum. A slice through the three-dimensional continuum solution at the center of the nozzle is shown as Fig. 11. Even though the outer boundary of the solution is beyond the continuum regime, it can be analyzed to find where flow transitions from continuum to rarefied. For the thruster plume, transition from continuum to rarefied flow is identified by a constant value of continuum breakdown parameter, $\mathrm{P} .{ }^{4}$ For the present analysis, a value of $\mathrm{P}=0.05$ is employed to produce the continuum breakdown surface.

In Figs. 11 and 12 the back shell of Phoenix, plume CFD solution, and continuum plume breakdown surface are illustrated. Note that the CFD solution is beyond the breakdown surface to show the extent of the CFD numerical domain. The continuum breakdown surface is now available for the next step in determining the aerodynamic changes caused by the RCS thruster firing, which is to provide a simulation of the rarefied flow field with and without the thruster. A triangulated geometry of the Phoenix outer mold lines

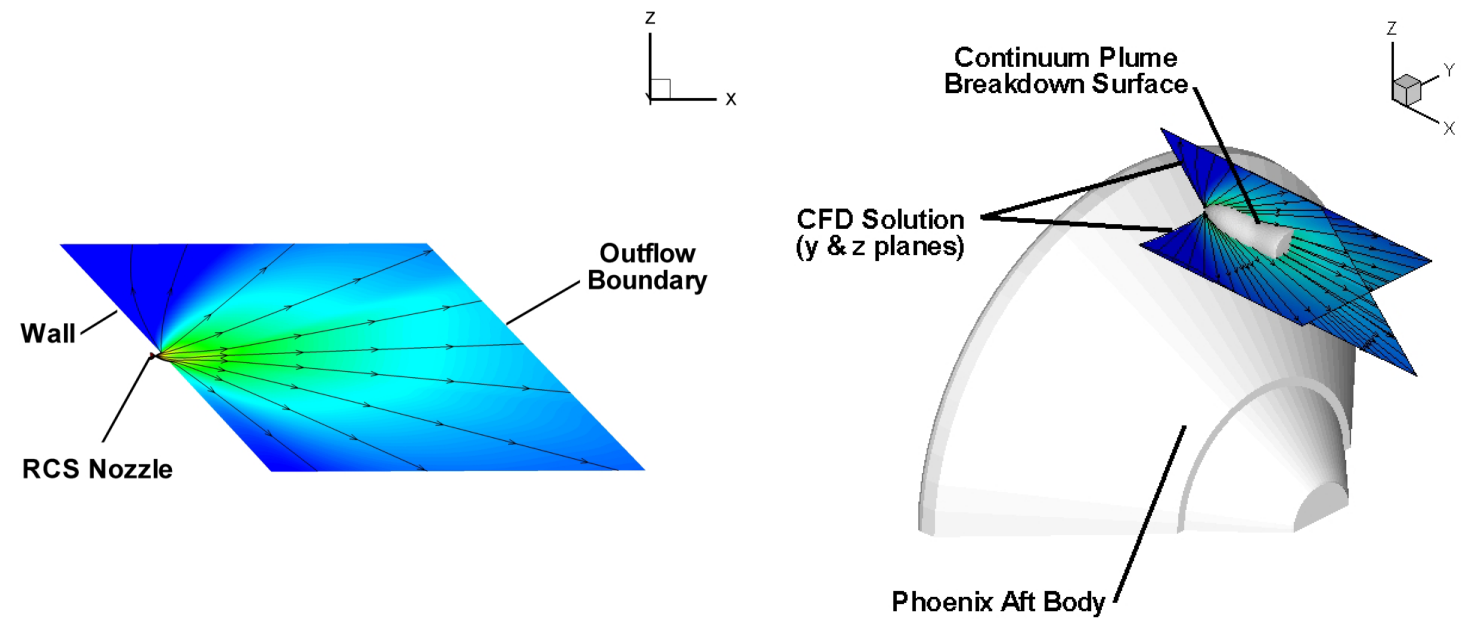

Figure 11. Thruster CFD Solution

Figure 12. Thruster continuum plume breakdown surface

and thruster continuum breakdown surface was created for a direct simulation Monte Carlo (DSMC) flow field calculation. For the breakdown surface, flow properties were interpolated from the CFD solution and assigned to the appropriate geometric node. The DSMC Analysis Code (DAC) ${ }^{5}$ was employed to produce the rarefied flow field. DAC is a robust software package that has many utilities, one of which easily allows 
geometric entities to be added as was done for this case with the RCS thruster as an inflow boundary. The simulation conditions were for a Mars atmosphere with $\mathrm{KnD}=0.1$ and $\beta=-6^{\circ}$, which has a free stream temperature of $166 \mathrm{~K}$, number density of $3.95 \times 10181 / \mathrm{m}^{3}$ molecules, velocity of $5520 \mathrm{~m} / \mathrm{s}$, mole fraction of $\chi(\mathrm{CO} 2)=0.95, \chi(\mathrm{N} 2)=0.03$, and $\chi(\mathrm{Ar})=0.02$. Two simulations were conducted at these conditions - one without the RCS thruster firing, as a baseline, and the other with the thruster firing as a comparator. The flow fields for the two simulations are shown as Figs. 13 and 14, respectively. With the continuum plume breakdown surface flow, the downstream wake density increases significantly (Compare Figs. 13 and 14).

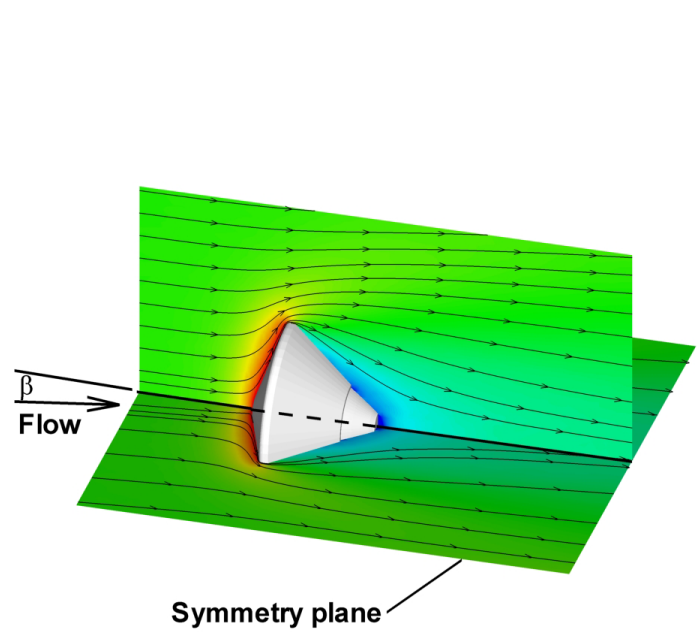

Figure 13. $\mathrm{KnD}=0.1$ flowfield, no thruster

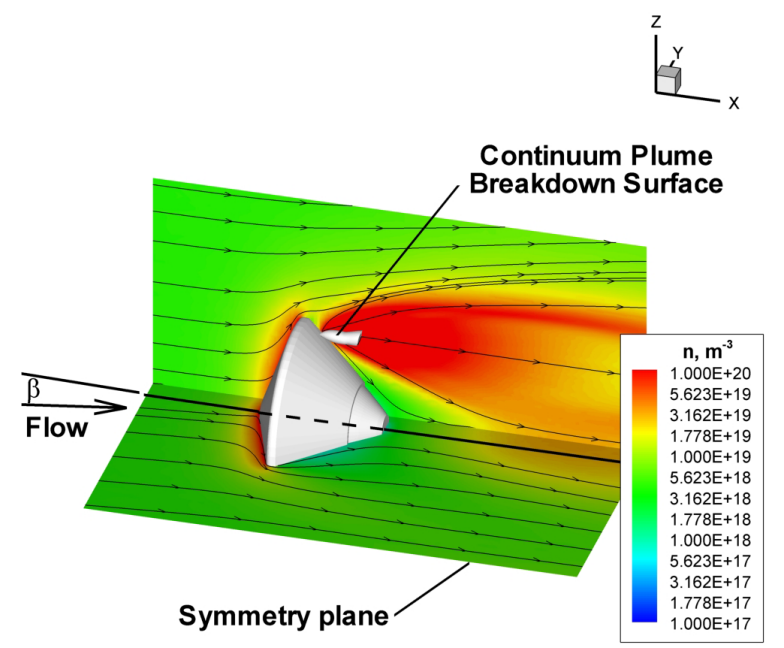

Figure 14. $\mathrm{KnD}=0.1$ flowfield, with thruster

\section{Hypersonic Regime}

Analysis in the hypersonic regime was carried out using Langley Aerothermodynamic Upwind Relaxation Algorithm (LAURA $)^{6,7,8}$ code. LAURA is a parallel three dimensional multi-block program that is extensively used in aerothermodynamic calculations of entry vehicles. LAURA can solve Euler, Thin Layer Navier-Stokes and full Navier-Stokes flow-fields using an upwind point- and line-implicit relaxation. The code can solve laminar and turbulent flows with and without thermo-chemical non-equilibrium. A wide range of chemical and thermal wall conditions are available.

In the present study an eight species Mars gas model $(\mathrm{CO} 2, \mathrm{CO}, \mathrm{C}, \mathrm{N} 2, \mathrm{~N}, \mathrm{NO}, \mathrm{O} 2, \mathrm{O})$ allows thermochemical nonequilibrium. Flow is laminar and the radiative equilibrium wall temperature condition with $\epsilon=$ .89 (representative of charred Super Lightweight Ablator (SLA)) is specified. A super-catalytic wall boundary condition is used, such that species concentrations near the wall are set to their free-stream values of $97 \% \mathrm{CO} 2$ and $3 \% N 2$ by mass. Computational grid for baseline (no thruster firings) calculations had 2.6 million points. This grid was modified to accommodate a thruster with use of Grid Morphing Software (MORPH) tool, developed at NASA Langley for the Shuttle Return to Flight (RTF) activities. The modified grid contained 5.9 million points. All solutions, generated for this analysis are symmetric about the $\alpha_{\text {total }}$ plane. This approach reduced the computational requirements. The interior of the thruster was not modeled. Instead, Chemical Equilibrium and Applications (CEA) program ${ }^{9}$ was used to determine nozzle exit conditions, given the nozzle area ratio and chamber pressure and temperature. The fuel is hydrazine $(\mathrm{N} 2 \mathrm{H} 4)$ and the products of its combustion are ammonia, hydrogen, nitrogen and traces of unspent hydrazine. CEA assumes equilibrium process through the convergent part of the nozzle, but from the throat to exit all reactions are frozen. Conditions computed at the nozzle exit are written into the modified CFD grid to emulate a thruster. In the CFD solution the thruster effluent is modeled as non-reacting ammonia.

Because of the large disparity between forces on the forebody and aftbody of the capsule during hypersonic flight, it is possible that numerical errors in evaluation of forebody moments will be comparable in magnitude to the RCS thruster-induced interference moment that is sought after. In other words, a small interference moment can be indistinguishable from numerical noise of the forebody solution. To isolate the desired information the moment summation is performed on the aftbody only. Algorithmically this means that the 
forebody solution is converged and frozen and becomes an input to the aftbody solution (wake).

The wake flow is unsteady, and the surface pressure distributions in the separated region oscillate with iteration. This oscillation results in an unsteady moment, which requires that the output be averaged over a number of iterations. The CFD iteration uses local time advance, but sometimes it is necessary to use global time advance to get through difficult transients in the wake. Physically, all of the wake should be advanced at the same rate using a global time step, but associated computational cost and a limited temporal accuracy of the code make this approach impractical. Instead, a local time step was used in this analysis with an understanding that unsteady processes within the wake evolve at their own rates, not necessarily consistent with each other. Figures 15 and 16 illustrate the unsteady moment output at a Mach 18.8 condition. The iteration history of the aftbody pitching moment about the center of gravity (CG) is shown. Figure 16 contains information from the Fig. 15, reduced by the Eq. (3). This step provides the direct measure of the effectiveness of the RCS thruster. Note that the scale is exaggerated, and a relatively small native RCS authority is illustrated.

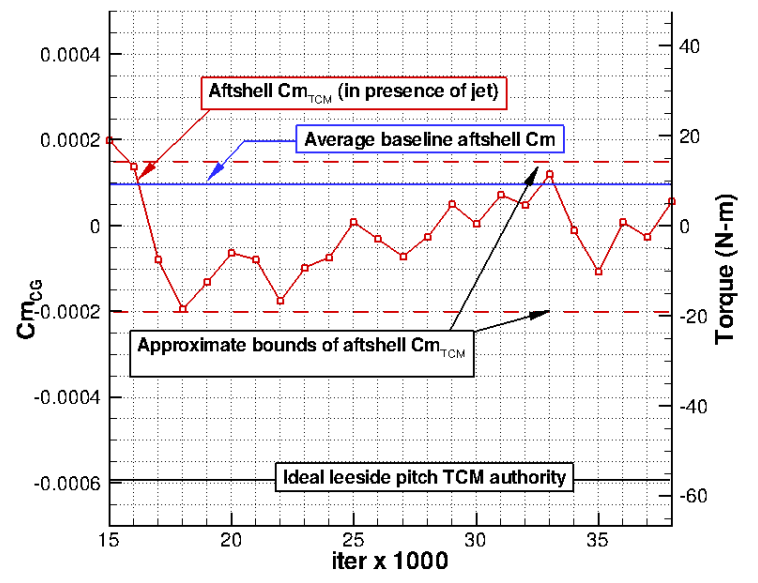

Figure 15. Iteration history of aftshell moment

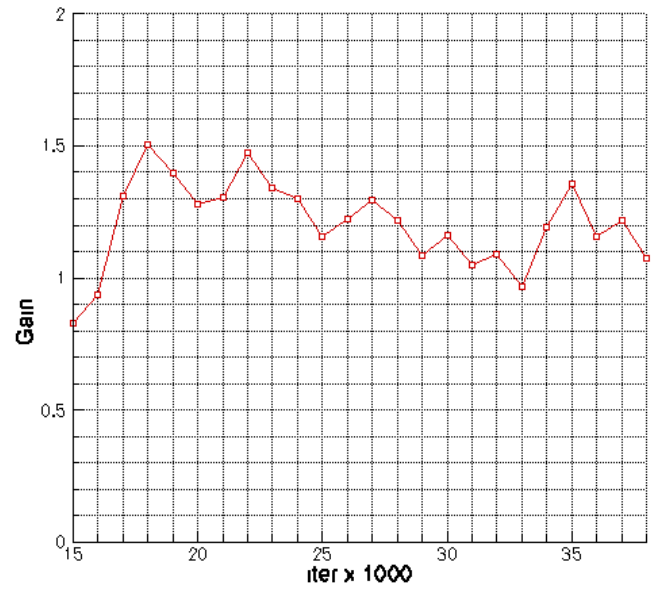

Figure 16. Iteration history of control gain

In the hypersonic regime calculations were performed at Mach 27.2 for yaw thrusters and Mach 18.8 for pitch and yaw thrusters. Mach 27.2 calculations included sideslip angles $\beta=6^{\circ}$ and $\beta=10^{\circ}$. Yaw thrusters were fired on the lee-side of the capsule. Solutions at Mach 18.8 were limited to angle of attack of $\alpha=10^{\circ}$ for pitch and an angle of sideslip $\beta=10^{\circ}$ for yaw thrusters.

\section{Supersonic Regime}

Supersonic flow regime computations were carried out using the unstructured Navier-Stokes solver FUN3D (Fully Unstructured Navier-Stokes in 3 Dimensions) with a steady-state laminar formulation of Roe's flux difference splitting scheme. RCS nozzle flows were computed from inlet to exit using a mass flux inflow boundary condition. Preliminary results were generated using calorically perfect air for both freestream and RCS thruster effluent on tetrahedral grids of approximately 3.5 million points. Additional solutions were run on a finer grid with approximately 7 million points, in addition to using a generic multispecies gas model, with two-component Mars atmosphere of $97 \%$ CO2, $3 \%$ N2, and RCS effluent modeled as $100 \%$ inert NH3. A matrix of cases were run at representative Mach 3 entry conditions as shown in the Table 1, with angle of attack sweeps for pitch and roll channel cases and yaw angle sweeps for yaw channel cases.

One point was also computed at a Mach 1.5 condition with 15 degree total angle of attack divided equally between pitch and yaw. Results obtained with the one-equation Spalart-Almaras turbulence model were also carried out which yielded nearly identical aerodynamic coefficients and are not presented here. 
Table 1. Run matrix for Mach 3 flight condition

\begin{tabular}{rr} 
RCS & attitude \\
\hline pitch & $\alpha=-8^{\circ},-4^{\circ},-2^{\circ}, 0^{\circ}, 2^{\circ}, 4^{\circ}, 8^{\circ}$ \\
roll & $\alpha=2^{\circ}, 4^{\circ}, 8^{\circ}$ \\
yaw & $\beta=-8^{\circ},-4^{\circ},-2^{\circ}, 0^{\circ}, 2^{\circ}, 4^{\circ}, 8^{\circ}$
\end{tabular}

\section{Results}

Computations of Phoenix RCS thruster efficacy were carried out in rarefied, hypersonic and supersonic regimes using the three techniques described above in detail. Results of these calculations are summarized in three respective sections, followed by a brief section on interpretation of these results.

\section{A. Rarefied Regime}

The effect of the thruster on back shell surface pressure coefficient is shown in Figs. 17 and 18. With the thrusters on, their effect on the back shell is local and confined to the region around them as shown in Fig. 18. However, the integrated effect between the thruster off and thruster on states results in a change in yaw moment coefficient to be insignificant (several orders of magnitude less than the native thruster authority). In addition, the yaw authority of $10.6 \mathrm{~N}-\mathrm{m}$ translates to a $\mathrm{Cn}=-0.168$ when compared to $\mathrm{Cn}$ of the RCS cases of -0.00242 . Therefore, sufficient yaw control authority is available to trim the vehicle at the $\mathrm{KnD}=$ 0.1 and $\beta=-6$ condition.

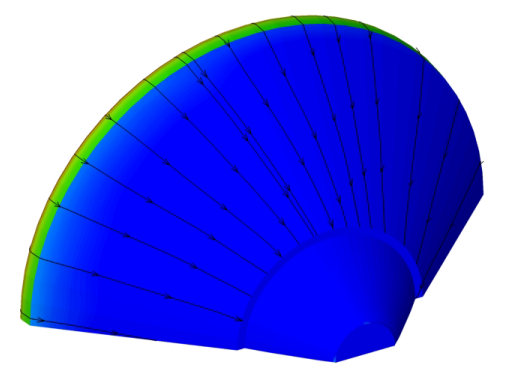

Figure 17. Surface pressure coefficient for $\mathrm{KnD}=0.1$ and $\beta=-6^{\circ}$ without thruster

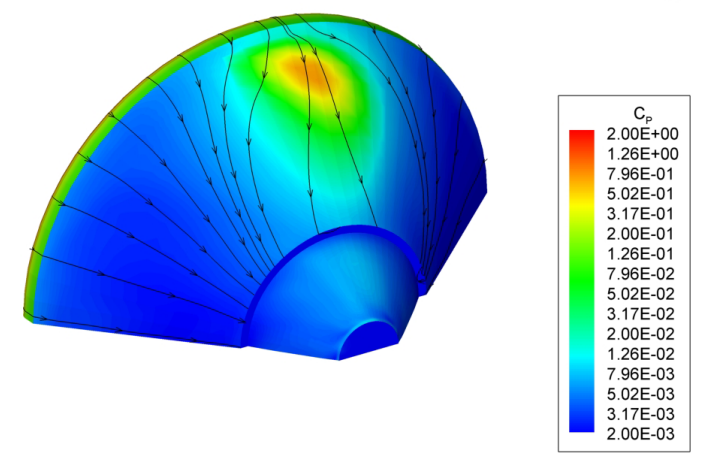

Figure 18. Surface pressure coefficient for $\mathrm{KnD}$ $=0.1$ and $\beta=-6^{\circ}$ with thruster

\section{B. Hypersonic Regime}

Figures 19-22 show computed surface pressures at Mach 27.2 with and without the thruster firings. Note the reduction of surface pressure and delayed separation when the thruster is on. It is hypothesized that this is happening due to entrainment of gas by the thruster plume, as it punches through the supersonic shear layer. Figure 23 shows the aftbody moment coefficients computed for the two angles of attack with and without the thruster firing. For reference, the nominal, or native yaw authority is plotted as a dashed line. The error bars do not indicate the level of uncertainty. Instead, they reflect only the variability of the moment output with iteration. Uncertainty would have to be added on top of this variation. As seen in the figure, the predicted interaction, or $C m_{\text {interference }}$ is significantly below the nominal authority. In other words the CFD solutions do not show a significant control degradation in yaw (or pitch, for that matter) at 
this high altitude flight condition. These results are in concurrence with the result of analysis in the rarefied regime, namely, unless aftbody pressure is high enough to produce an appreciable torque on the capsule, it is highly unlikely that disturbing the wake would result in significant levels of interference torque.

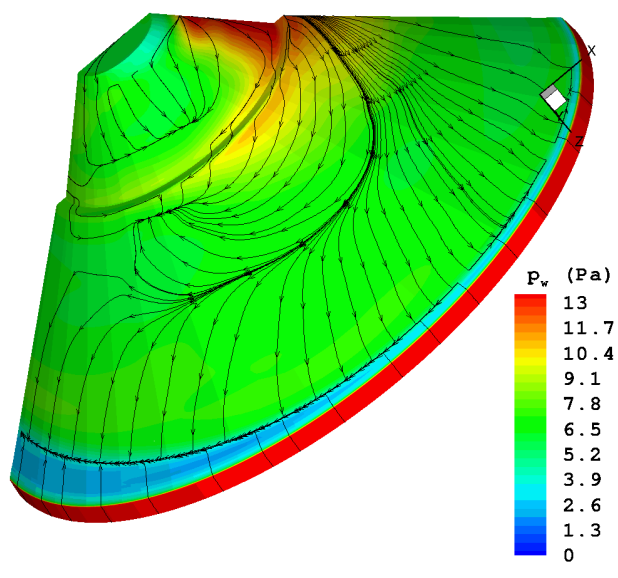

Figure 19. Surface pressure for Mach 27.2 and $\beta=6^{\circ}$ without the thruster

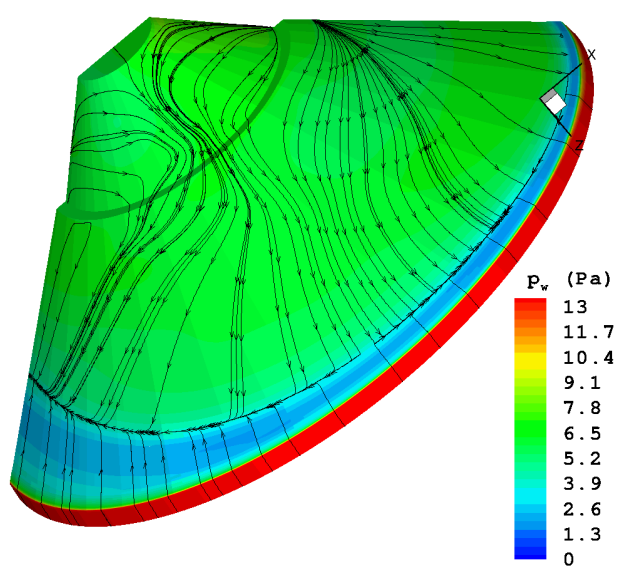

Figure 21. Surface pressure for Mach 27.2 and $\beta=10^{\circ}$ without the thruster

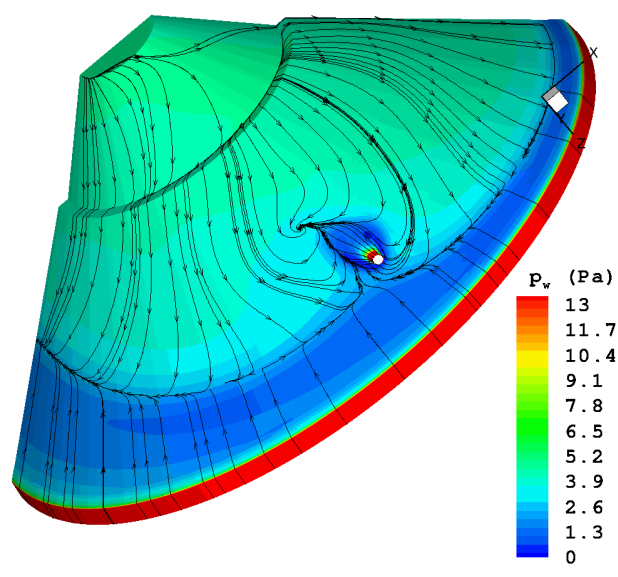

Figure 20. Surface pressure for Mach 27.2 and $\beta=6^{\circ}$ with the thruster

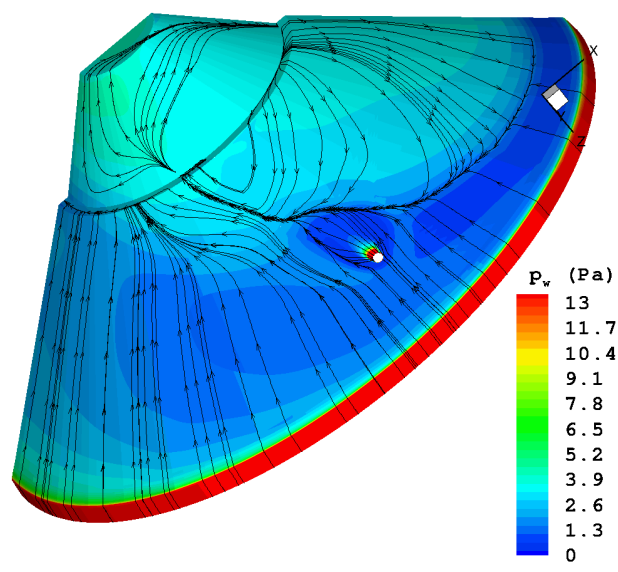

Figure 22. Surface pressure for Mach 27.2 and $\beta=10^{\circ}$ with the thruster

Figures 24 and 25 show pitch and yaw control authority on top of the static stability curve at Mach 18.8, which corresponds to the peak of dynamic pressure on the trajectory. As the figures indicate, RCS is not effective at changing the attitude of the capsule at this Mach number. Pitch thrusters are capable of about $0.5^{\circ}$ change in attitude. Yaw thrusters, due to lower native authority of $10.5 \mathrm{Nm}$, can only produce about $0.1^{\circ}$ change in the sideslip angle. As Figs. 26 and 27 indicate, the native moment is comparable to the variability of aftshell CFD solutions. Plots show nominal authority, baseline aftshell moment and the aftshell moment, perturbed by the flow due to interaction with the thrusters. Figure 26 indicates that the thruster on the lee-side $\left(\alpha=-10^{\circ}\right)$ of the capsule results in favorable difference in authority (i.e. $C m_{\text {interference }}=C m_{T C M}-C m_{\text {Baseline }}$ has the same sign as the nominal RCS thruster authority moment), while the thruster on the windside $\left(\alpha=10^{\circ}\right)$ results in an adverse effect $\left(C m_{\text {interference }}\right.$ is opposing nominal authority). Solution with the leeside yaw thruster (Fig. 27) shows an adverse interference torque. Again, no uncertainties are applied to these calculations, and error bars only indicate the solution variability with iteration. Because yaw thruster authority is so small in comparison to both the solution variability and the interference moment it can not be said with any certainty that the RCS will perform acceptably during flight near peak dynamic pressure. 


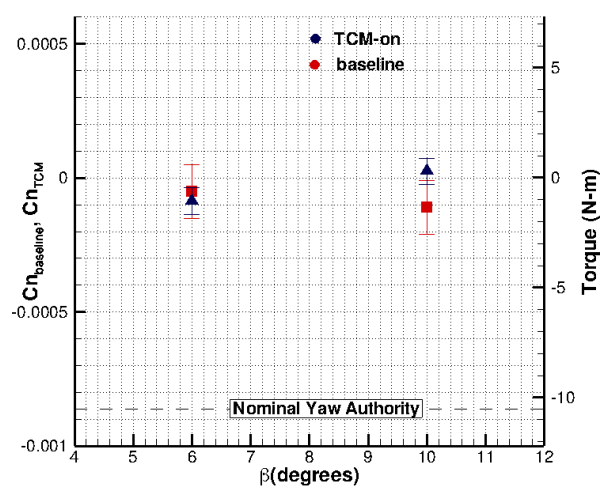

Figure 23. Yaw interaction at Mach 27.2 for $\beta=6^{\circ}$ and $10^{\circ}$

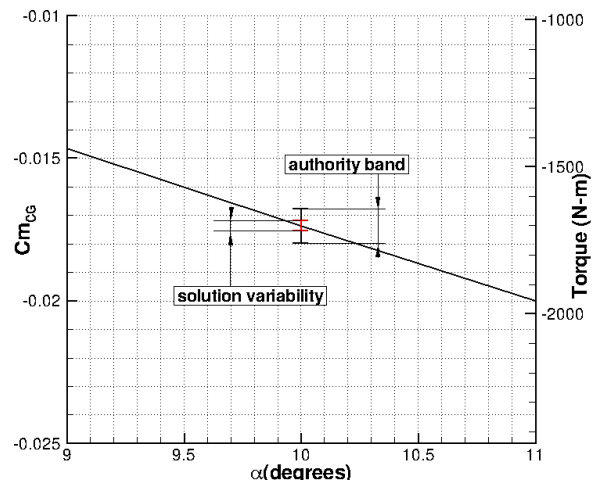

Figure 24. Static stability, pitch authority and solution variability at Mach 18.8 near $\alpha=10^{\circ}$

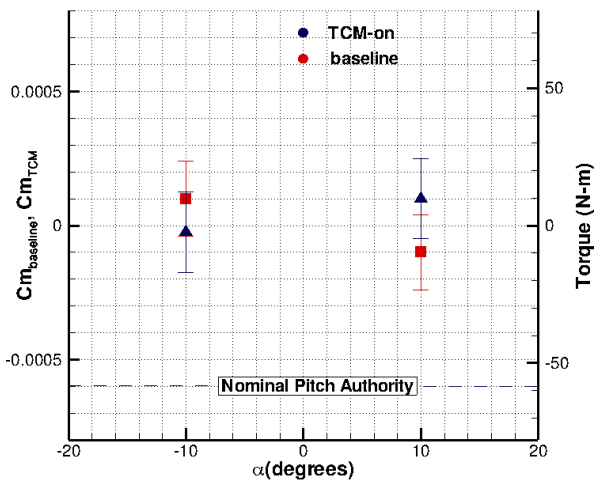

Figure 26. Pitch interference at Mach 18.8, $\alpha= \pm 10^{\circ}$

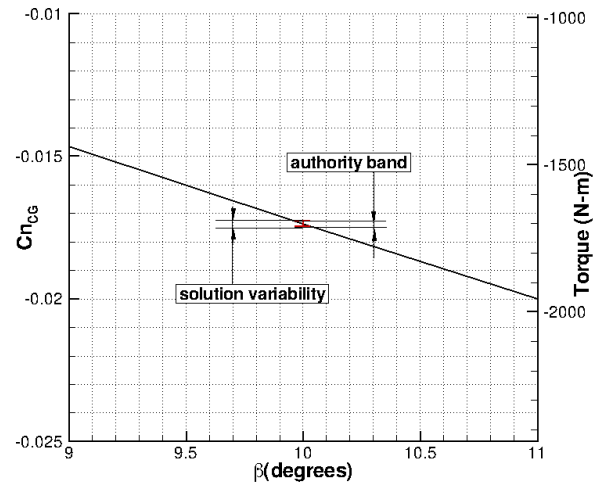

Figure 25. Static stability, yaw authority and solution variability at Mach $\mathbf{1 8 . 8}$ near $\beta=10^{\circ}$

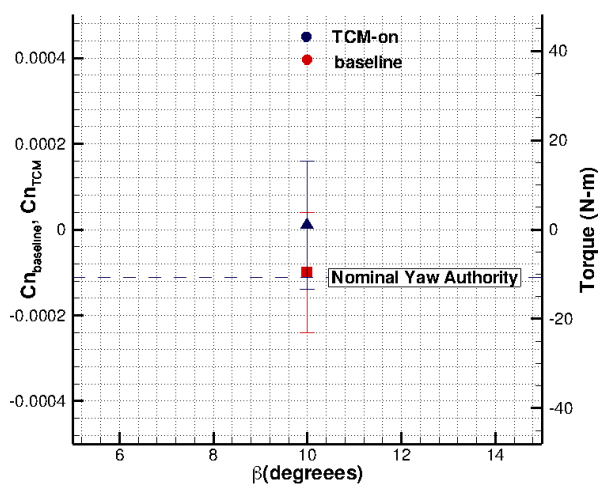

Figure 27. Yaw interference at Mach 18.8, $\beta=10^{\circ}$

\section{Supersonic Regime}

Due to the unsteadiness of the wake flow a large degree of solution variability was experienced. Hence, mean aerodynamic coefficients were approximated by averaging over an interval of at least ten thousand iterations. 
In the accompanying figures, error bars denote solution variability only, and do not include any uncertainty, which would still need to be added enlarging the error bars further.

For the pitch channel cases, RCS firings were assigned to induce a nose-down reaction (i.e., a negative pitching moment coefficient). Pitch channel cases were run at seven angles of attack as shown in Fig. 28. For each solution, the mean interaction coefficient (here and further labeled $\Delta \mathrm{C}_{m}$ for pitch and $\Delta \mathrm{C}_{n}$ for yaw, and defined as shown previously in Eq. (2)) and solution variability are depicted. As seen, a control authority deficit was observed in all pitch channel cases, except for the $-4^{\circ}$ angle of attack case which marginally crossed into surplus control authority. However, in all cases, solution variability was sufficiently large to make a deficit condition appear likely. That is, if RCS thrusters were fired to produce the requested commanded moment (e.g., $46 \mathrm{Nm}$ ), the interaction between aerodynamic flowfield and thruster plume would result in a moment less than that requested. These results indicated that net pitch control authority could be degraded significantly (upwards of $80 \%$ ) due to the aero/RCS interaction. Solutions with the finer grid and generic gas path model yielded very similar results.

The mechanism responsible for loss of control authority in these cases was the RCS thruster's plume entrainment of wake flow from the RCS thruster side of the capsule, forcing the majority of recirculation to occur towards the opposite side of the capsule. This concentration of upstream-traveling wake flow can be seen in Fig. 29 to the side of the backshell opposite the RCS plume flow.

Additional runs at $\pm 20^{\circ}$ angle of attack showed that the pitch channel aero/RCS interaction subsides at large angles of attack. At these two points, maximum pitch control authority degradation was approximately $11 \%$, leading to the conclusion that pitch RCS authority was sufficiently available at these conditions.

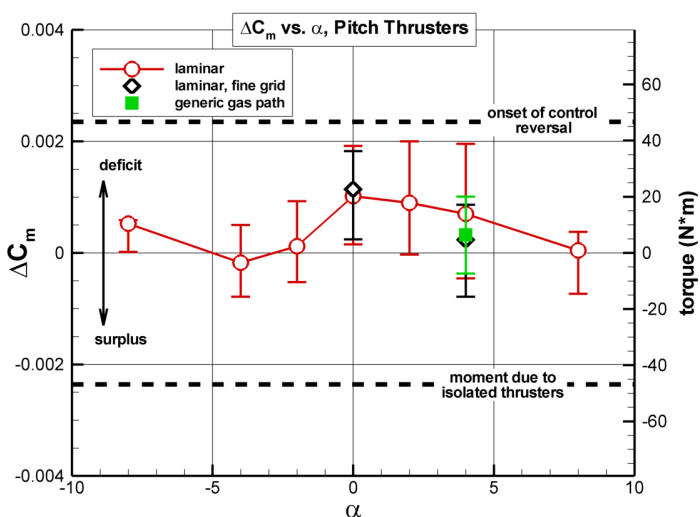

Figure 28. Interaction in pitch at Mach 3

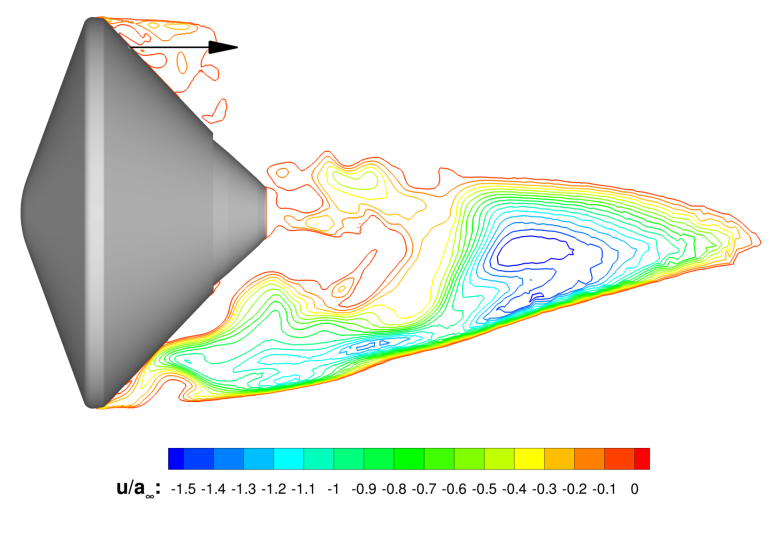

Figure 29. Recirculating flow in the pitch plane concentrating to the side of the capsule opposite RCS jet (arrow) at $\alpha=0^{\circ}$

For the yaw channel cases, RCS thruster firings were assigned to induce a negative yawing moment coefficient. Seven side-slip angle solutions were run in the yaw channel as shown in Fig. 30. For each solution, the mean coefficient and solution variability are depicted. As seen, the predicted steady-state interaction coefficients in the yaw channel were of such magnitude and sign as to suggest a control reversal at $0^{\circ}$ and $2^{\circ}$ incidence. That is, although a commanded moment was requested having a specific magnitude and sign, a moment in the opposite direction would result. In addition, solution variability was quite large for all incidence angles and far in excess of available yaw control authority.

Roll channel RCS cases revealed no primary interaction about the roll axis, as was expected due to the inherent axisymmetry of the configuration. However, strong secondary interactions were induced about the pitch and yaw axes, due to roll RCS thruster plumes sweeping tangentially across the backshell and rearranging large regions of wake flow as shown if Figs. 31 and 32. The worst-case interaction of roll RCS thrusters about the pitch axis was approximately $70 \%$ of the pitch control authority, while about the yaw axis accounted for nearly twice the yaw control authority. Variability in these solutions was greater than the available authority in either of the respective control channels.

A single solution was also run at a Mach 1.5 entry condition at a $15^{\circ}$ total angle of attack, rotated $45^{\circ}$ about the body axis, so as to determine whether pitch channel RCS thruster firings alone could be used 


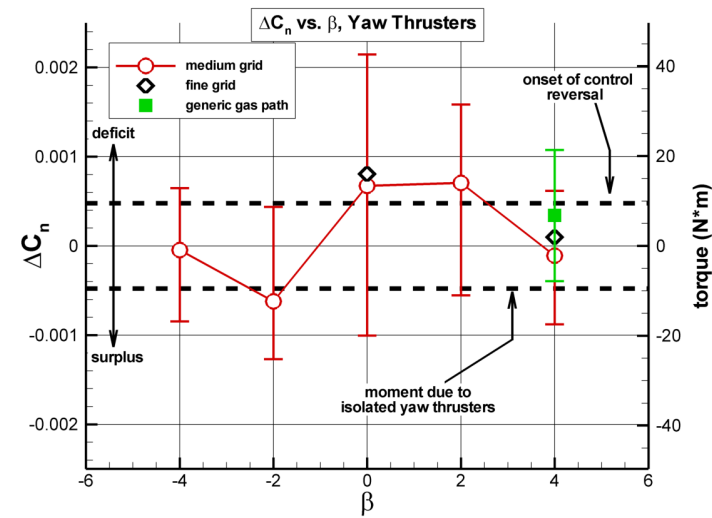

Figure 30. Interaction in yaw at Mach 3. Note apparent control reversal at $\beta=0^{\circ}$ and $4^{\circ}$

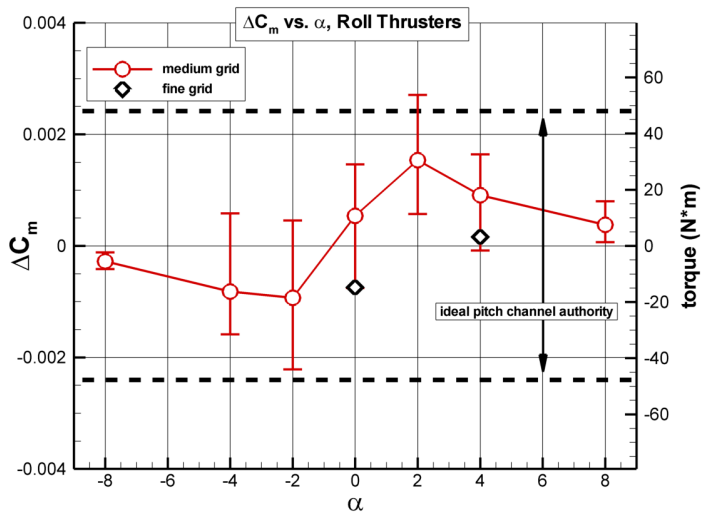

Figure 31. Interaction of roll RCS about pitch axis

as a substitute for yaw channel control. Taking unsteady effects into account, however, the cross-channel interaction about the yaw axis again exceeded the available yaw control authority by a factor greater than four (see figure 33).

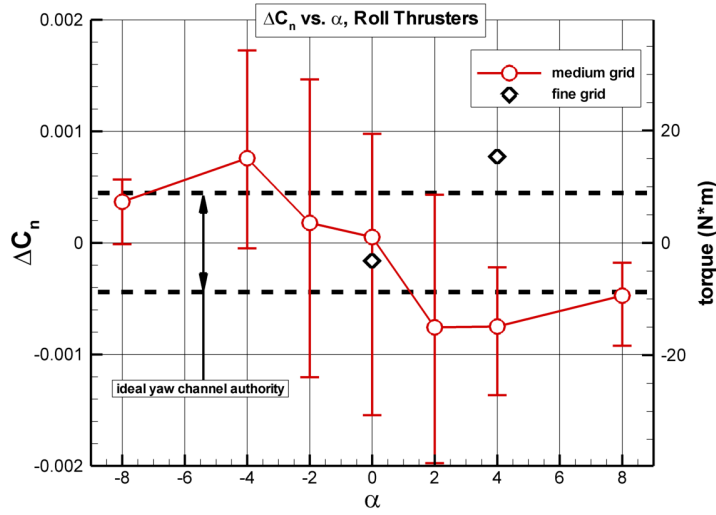

Figure 32. Interaction of roll RCS about the yaw axis

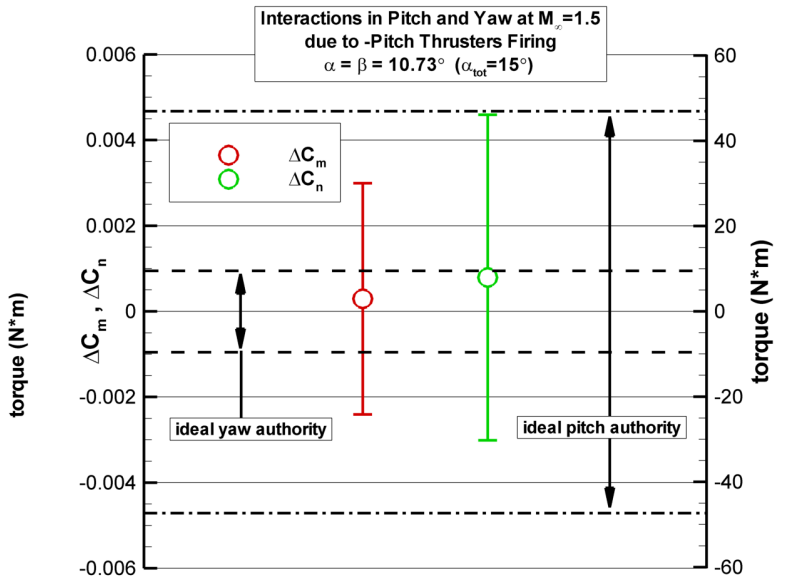

Figure 33. Interaction of pitch RCS about pitch and yaw axes, with $\alpha=\beta=10.73^{\circ}$

\section{Overview}

Results presented in the preceding sections suggest that the Phoenix RCS system may not be able to effectively control the capsule in every flight regime due to sugnificant interaction between the aerodynamic flowfield and thruster plumes. Calculations in rarefied and high hypersonic regimes indicate that problems there are unlikely, mainly because of low aftbody pressures. There is simply not enough pressure to generate a significant interference moment. Computations at a lower hypersonic point near peak dynamic pressure and at a supersonic Mach 3 condition suggest significant control authority degradation in the pitch channel and a possible reversal in the yaw channel. Solutions for roll thrusters indicated strong cross-coupling into pitch and yaw channels which overwhelms the available yaw control authority. These results are given prior to adding any uncertainty. Because of this, there is little confidence in the effectiveness of the Phoenix RCS during hypersonic and supersonic flight, moreover, a possibility exists of a control reversal. 


\section{Conclusion}

Numerical analyses of the efficacy of the Phoenix Reaction Control System (RCS) showed that the system might be inadequate to control the capsule during entry due to a significant interaction between the wake and the plumes of the RCS thrusters. Specifically, significant control degradation in pitch and control reversal in yaw may occur near peak dynamic pressure and at supersonic speeds. In addition, significant cross-coupling into pitch and yaw channels due to use of roll thrusters during supersonic flight is predicted. Based on the results it can not be said with any certainty that the Phoenix RCS system will perform adequately during most of the continuum regime of the atmospheric entry. The techniques utilized in these analyses have known issues. Calculations assume a thruster that is constantly on due to the limitations of current state-of-the-art techniques. However, it is believed that the present approach bounds the phenomena. Similarly, because of the complexity of the capsule wake it is not practical to attempt to achieve grid independence. Increasing the grid level was found to produce a non-monotonic response of the moment output.

In summary, the control authority of the Phoenix RCS system is low, particularly in the yaw channel. At some flight conditions the RCS control moment is lower then the level of unsteadiness in a baseline Computational Fluid Dynamics (CFD) solution. This illustrates that present CFD techniques are at or beyond their limit when analyzing RCS control authority with such a low native moment. It also illustrates that it is beneficial to design an RCS system with greater native moment capability, so that these problems can be avoided. Because of the issues identified through the present analyses, the Phoenix Project changed its plan regarding the use of the RCS system during atmospheric entry. Specifically, the RCS system deadbands were sufficiently widened for flight through the continuum regime as to essentially eliminate any thruster firings. Consequently, Phoenix became the first ballistic uncontrolled non-spinning entry.

\section{Acknowledgments}

Authors would like to thank Peter A. Gnoffo and Pieter G. Buning of NASA Langley Research Center and Michael J. Wright of NASA Ames Research Center for consultations and discussions that were instrumental to this work.

\section{References}

\footnotetext{
${ }^{1}$ Gnoffo P., Planetary-Entry Gas Dynamics, Annu. Rev. Fluid Mech., 1999.31:459-94

${ }^{2}$ Edquist K. T., Desai P. N., and Schoenenberger M., Aerodynamics Prediction for the Mars Phoenix Entry Capsule, AIAA Paper 2008-7219, Proceedings of AIAA Guidance, Navigation, and Control Conference, Honolulu, HI, August 2008.

${ }^{3}$ AeroSoft, GASP Version 4.0 User's Manual, ISBN 09652780-5-0, 2002

${ }^{4}$ Bird, G.A., Molecular Gas Dynamics and the Direct Simulation of Gas Flows, Oxford University Press, Oxford, 1994

${ }^{5}$ LeBeau G. J. and Lumkin F. E. III, Application Highlights of the DSMC Analysis Code (DAC) Software for Simulating Rarefied Flows, Computer Methods in Applied Mechanics and Engineering, 191, No. 6-7, 595-609, 2001

${ }^{6}$ Gnoffo P. A., Gupta R. N., and Shinn J. L., Conservation Equations and Physical Models for Hypersonic Air Flows in Thermal and Chemical Non-equilibrium, NASA TP-2867, February 1989.

${ }^{7}$ Gnoffo P. A., An Upwind-Biased, Point-Impliscit Algorithm for Viscous, Compressible Perfect-Gas Flows, NASA TP2953, February 1990.

${ }^{8}$ Cheatwood, F. M. and Gnoffo, P. A., Users Manual for the Langley Aerothermodynamic Upwind Relaxation Algorithm (LAURA), NASA TM-4674, April 1996.

${ }^{9} \mathrm{McBride}, \mathrm{B}$. J. and Gordon S. Computer Program for Calculation of Complex Chemical Equilibrium Compositions and Applications, Part II - User Manual and Program Description, NASA RP-1311, 1996

${ }^{10}$ Schoenenberger M., Cheatwood F. N. and Desai, P. N., Static Aerodynamics of the Mars Exploration Rover Entry
} Capsule, AIAA 2005-56 